\title{
CHIRP-FREE TRANSMISSION THROUGH A NOLM BASED OPTICAL REGENERATOR
}

\author{
K.Sponsel ${ }^{1}$, M.Meissner ${ }^{1}$, K.Cvecek ${ }^{1}$, B.Schmauss ${ }^{2}$, G.Leuchs ${ }^{1}$ \\ ${ }^{1}$ Institute of Optics, Information and Photonics, University Erlangen-Nuremberg \\ Guenther Scharowski Str.1, 91058 Erlangen, Germany \\ ${ }^{2}$ Electrical Engineering Department, University of Applied Science Regensburg \\ Prüfeninger Str.58, 93049 Regensburg, Germany
}

\begin{abstract}
We experimentally show that a strongly asymmetric nonlinear optical loop mirror (NOLM) based 2-R regenerator does not degrade the pulse quality in a fiber optic transmission line. The signal at the NOLM output port shows only negligible chirp if a fundamental soliton propagates in the strong arm of the NOLM. This behavior is measured for splitting ratios of 91:09 and 85:15. The NOLM does not insert penalties regarding the pulse shape and is therefore suitable for inline regeneration of optical transmission lines.
\end{abstract}

\section{INTRODUCTION}

In optical communication at high bitrates optical regeneration $[1,2]$ is a major issue of current reasearch to reduce costs and extend system reach. Though the focus presently lies on optical 3-R regenerators, 2-R regenerators become more and more interesting. They can offer a cost saving alternative as retiming is not always needed. An example of a 2-R type regenerator is the asymmetric NOLM first suggested by Doran and Smith [3]. It is capable of considerably reducing the amplitude noise introduced by amplifiers on the "1" bit and thus improving the BER and the bitrate distance product [4]. An amplifier noise reduction of $12 \mathrm{~dB}$ on the " 1 " bit has already been shown [5]. But for the use as an inline regenerator in fiber optical transmission lines not only the reduction of amplitude jitter is of interest. At high bitrates of $40 \mathrm{GBit} / \mathrm{s}$ and more, the pulse quality is of major importance. In particular, the low chirp tolerance of such systems will be a major problem. For the investigation of pulse quality Frequency Resolved Optical Gating (FROG) [6] is a powerful technique, as it provides a complete description of the electrical field envelope and phase of 
the pulses as a function of time. In this paper we use the FROG technique to analyse the pulse quality at the output port of a highly asymmetric NOLM with special emphasis on chirp.

\section{PRINCIPLE OF OPERATION OF THE NOLM}

The NOLM represents a fiber Sagnac interferometer, consisting of a fiber coupler and a fiber loop connected to the two output ports of the coupler, as shown in Fig.2. An incoming signal pulse is split into two counter-propagating pulses with different peak powers, according to the splitting ratio. Because of the Kerr nonlinearity in the fiber the two pulses gather different nonlinear phase shifts, depending on their different peak powers, and interfere at the coupler after one roundtrip. As this interference is power dependent it leads to a nonlinear input output power transfer characteristic that depends on the splitting ratio, as displayed in Fig.1.

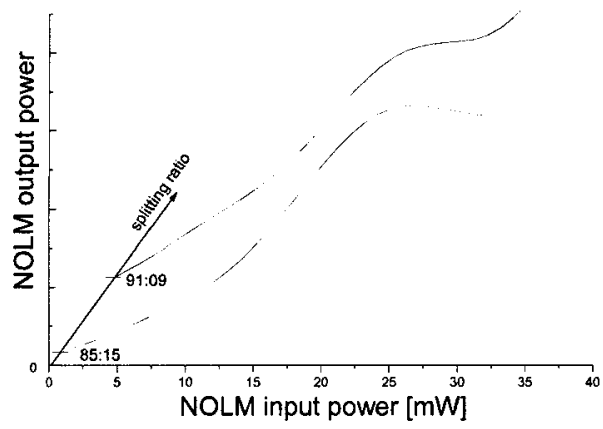

Figure 1. NOLM power transfer characteristics for splitting ratios of 91:09 and 85:15. For more symmetric splitting ratios, higher input powers are needed to get into the plateau region.

With the used splitting ratios of 85:15 and 91:09 regions with low slopes are present in the the NOLM characteristics. This regions between the points of slope smaller than one are called plateau regions. For the given setup and a splitting ratio of 91:09 the plateau is located between input powers of $22.5 \mathrm{~mW}$ and $27.5 \mathrm{~mW}$, corresponding to peak powers of $750 \mathrm{~W}$ and $918 \mathrm{~W}$. Amplitude noise reduction is achieved if an incoming noise distribution is located on the plateau region, as its width is significiantly reduced when transfered to the output port [5]. Best noise reduction is possible if the noise distribution is centered around the middle of the plateau. With decreasing optical signal to noise ratio (OSNR) the noise distribution broadens. Therefore the splitting ratio of the 
NOLM has to be adapted to the input OSNR, because the width of the plateau region increases when the splitting ratio becomes more symmetric. For an OSNR better than $44 \mathrm{~dB}$ a ratio of 91:09 is needed and for an OSNR of $28 \mathrm{~dB}$ a ratio of 85:15 [7]. Optimal noise reduction performance is observed around an OSNR of 30dB. The splitting ratios of 91:09 and 85:15 in our experiment are chosen to investigate the OSNR interval, where the NOLM is operating as a regenerator. [8].

\section{EXPERIMENTAL SETUP}

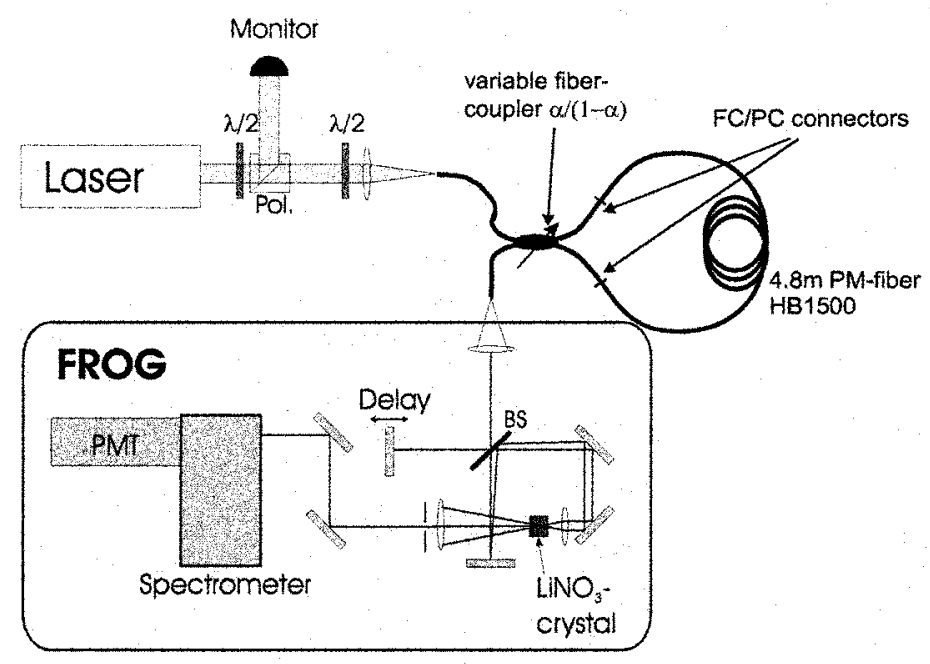

Figure 2. Experimental setup. The input polarization is matched by a half wave plate to one of the principle axis of the polarization maintaining NOLM fiber. The splitting ratio of the NOLM can be adjusted by the variable fiber coupler.

The experimental setup is shown in Fig.2. The laser source is a mode-locked $\mathrm{Cr}^{4+}: Y A G$ laser emitting pulses of sech-shape with $162 \mathrm{fs}$ pulse duration at $1495 \mathrm{~nm}$ and a repetition rate of $163 \mathrm{MHz}$. As we use soliton transmission within the NOLM, the results are transferable to optical communication systems where e.g. $1.5 \mathrm{ps}$ are used for $160 \mathrm{GBit} / \mathrm{s}$. Neglecting higher order effects as the stimulated Raman effect the properties of solitons such as self stabilization and pulse shaping are independent of the fiber type and pulse length [9]. The drawback is, that for longer pulse durations much longer fiber loops or specially designed photonic crystal fibers [10] are needed. To reach the plateau region the phase differences $\Delta \Phi$ due to the nonlinear Kerr effect of the two counterpropagating pulses in the NOLM must be about $1.5 \pi$. For a fundamental soliton in the intense arm of the NOLM the required loop length 
for plateau formation is then given by equation 1. In units of dispersion length $L_{D}$ the fiber loop length depends only on the splitting ratio $\alpha$ and not on the pulse duration [9].

$$
L=L_{D} \frac{\Delta \Phi}{|1-2 \alpha|}
$$

In our experiment the optical power of the laser beam is adjusted with a half wave plate and a polarizing beam splitter at the NOLM input. The polarization of the signal is matched to one of the principal axis of the NOLM fiber by a second half wave plate. The NOLM comprises a tunable fiber coupler consisting of polarization maintaining HB1500 fiber and 4.9m fiber loop of the same type of fiber. The length of the loop is chosen to aquire the $1.5 \pi$ phase shift for the plateau region with a soliton in the intense arm at a splitting ratio of 91:09, according to equation 1. The NOLM output pulses are analysed with the FROG, consisting of a Michelson interferometer based SHG autocorrelator and a monochromator to spectrally resolve the SHG signal. Finally the signal is detected by a photomultiplier at the spectrometer output as a function of wavelength and time.

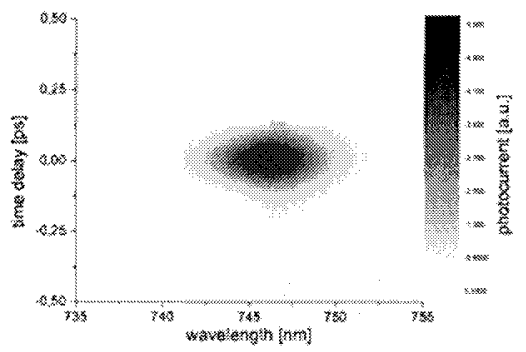

Figure 3. a) Experimental FROG trace of the NOLM output pulse, taken at an input peak power of $835 \mathrm{~W}$ and a splitting ratio of 91:09.

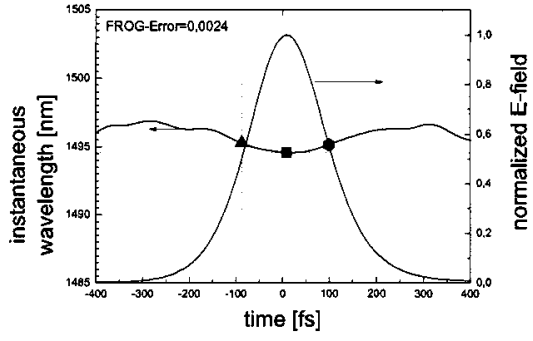

Figure 4. b) Electrical Field (right axis) and instantaneous wavelength (left axis) derived from the FROG trace in Fig.3 The dip in the pulse center originates from a slight quadratic chirp of the $\mathrm{Cr}^{4+}: Y A G$ Laser

The FROG traces were taken for several input powers along the nonlinear transfer characteristic. These traces were recorded with a resolution of 128 steps in the time domain and 512 steps in the spectral domain and were corrected for the spectral response of the photo multiplier. The high resolution in the spectral domain allows for a simple method for noise reduction of the 
signal by averaging over 4 pixels in the spectrum. Therefore, for the signal retrieval FROG traces of 128 times 128 pixels were used, as shown in Fig.3. From each FROG trace the electrical field envelope and the phase were calculated as functions of time as shown in Fig.4. Differentiating the phase the instantaneous frequency can be calculated [6] and converted into an instantaneous wavelength, which is more convenient. To quantify the chirp of the transmitted pulses, we take the instantaneous wavelength at the points of half the maximum of the electrical field envelope and at the pulse center, shown in Fig.4.

\section{EXPERIMENTAL RESULTS}

To investigate the pulse quality behind the NOLM for lower OSNRs, like $28 \mathrm{~dB}$ we chose a splitting ratio of $85: 15$. The results are presented in Fig.5. The input-output characteristic shows a flat plateau region which starts around an average input power of $25 \mathrm{~mW}$. The instantaneous wavelength at the FWHM and in the pulse center of the E-field are plotted versus the NOLM input power in the left hand side of Fig.5.
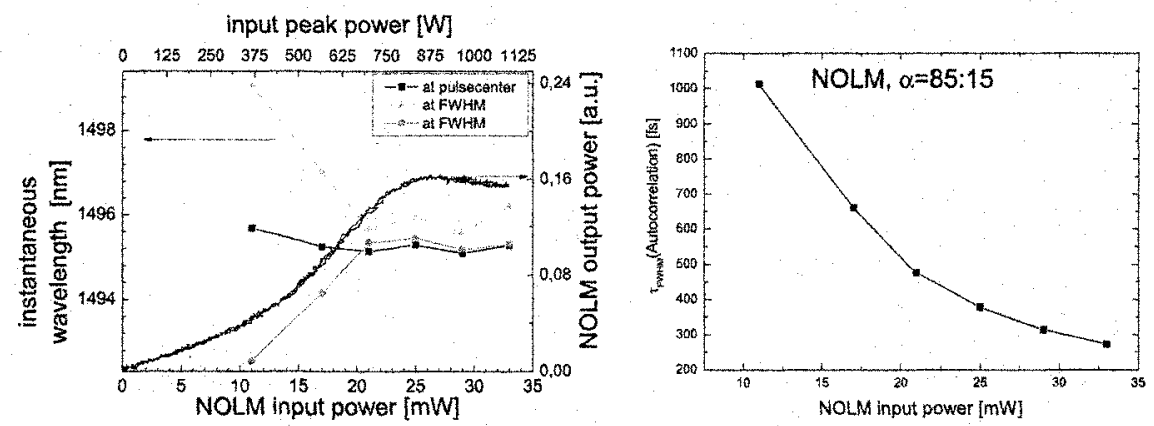

Figure 5. NOLM characteristic and development of the instantaneous wavelength at a splitting ratio of 85:15 (left), and autocorrelation width of the transmitted pulses versus input power (right)

Input powers below $20 \mathrm{~mW}$ lead to dispersive pulse broadening and therefore a linear chirp, which can be seen in the autocorrelation and the instantaneous wavelength in Fig.5. The instantaneous wavelengths of the pulse edges are about $2 \mathrm{~nm}$ away from the center wavelength. Increasing the input power leads to soliton formation in the strong arm of the interferometer and causes a reduction of chirp of the output pulse. The pulse length decreases. To match the power of the fundamental soliton in the intense arm an average NOLM in- 
put power of $26.5 \pm 1.5 \mathrm{~mW}$, corresponding to a peak power of about $885 \mathrm{~W}$ is needed. In this case the maximum difference of the instantaneous wavelength within the FWHM of the E-field of the pulse is below $0.65 \mathrm{~nm}$. This is only a small fraction of the pulse spectral width of $\Delta \lambda_{F W H M}=24.2 \mathrm{~nm}$ in the electrical field, implying that the pulses after the NOLM are nearly free of chirp. The power range for chirp-free NOLM output pulses stretches from $20 \mathrm{~mW}$ to about $30 \mathrm{~mW}$, covering the range of input powers in the plateau region of the nonlinear transfer characteristic which is used for noise reduction. Note that even the intrinsic chirp of the laser, resulting in a wavelength difference between the pulse edges and the center of each laser pulse of $2.7 \mathrm{~nm}$ is reduced. The lasers quadratic chirp originates from its internal group velocity dispersion compensation. The end of the plateau region could not be reached due to the experimental limitation in the maximum available laser power.
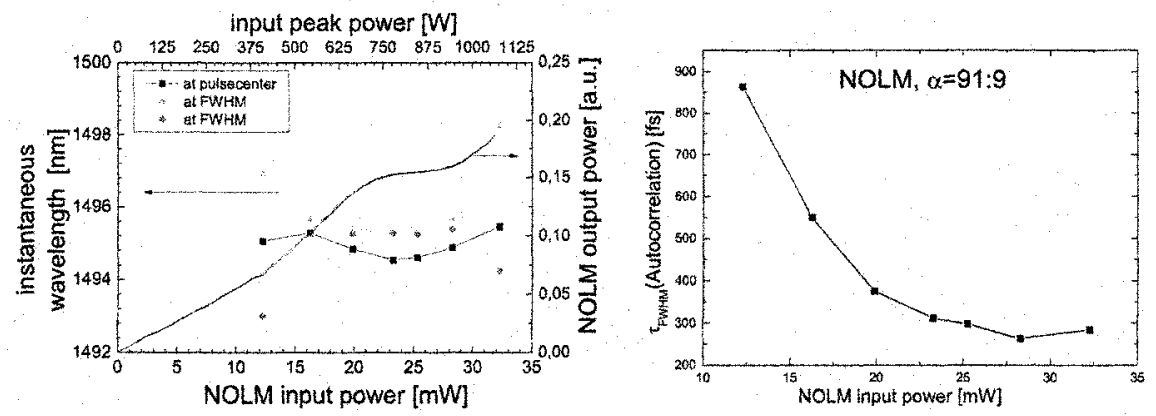

Figure 6. NOLM characteristic and development of the instantaneous wavelength at a splitting ratio of 91:09 (left), and autocorrelation width of the transmitted pulses versus input power (right)

The nonlinear input output characteristic for a splitting ratio of 91:09 is depicted in Fig.6. The fundamental soliton was measured at an average input power of $23 \pm 1.5 \mathrm{~mW}$. Accordingly at the given splitting ratio, an input power of $25 \pm 1.5 \mathrm{~mW}$ is needed to launch a soliton into the intense arm of the NOLM, corresponing to a peak power of about $835 \mathrm{~W}$. For an average NOLM input power of $25 \mathrm{~mW}$ and a splitting ratio of 91:09 electrical field and instantaneous wavelength are presented in Fig.4. As expected from a soliton the change of chirp is quite similar to the case with a splitting ratio of 85:15 shown in Fig.5. For input powers close to $25 \mathrm{~mW}$ the output pulses are practically free of chirp. When the input power exceeds the fundamental soliton power in the strong arm above $28.5 \mathrm{~mW}$, the chirp of the pulse increases again. This is due to effects like soliton formation with emission of dispersive waves and changes in the 
interference as the weak pulse is stronger affected by the Kerr-effect. But here the NOLM input output characteristic has a slope larger than unity, thus this part is not suitable for noise reduction and is therefore of no practical interest.

\section{DISCUSSION}

For an optimal pulse transfer through the NOLM the propagation of a fundamental soliton in the intense arm of the interferometer is used. Fundamental soliton are self stabilising, have a constant phase and are thus free of chirp. In the weak arm of the interferometer the pulse is broadened in time by dispersion. At the fiber coupler the soliton in the strong arm interferes and recombines with the dispersive pulse of the weak arm. But as the weak pulse added has only $11 \%$ of the soliton power for a splitting ratio of $91: 09$, and $17.6 \%$ for a splitting ratio of $85: 15$ the perturbation of the soliton pulse shape is small and the soliton properties are mostly maintained. Hence also in this case the NOLM output pulse is practically free of chirp.

\section{SUMMARY}

We have demonstrated that a NOLM type 2-R regenerator does not introduce chirp or changes in the pulse shape in an extended region around its point of operation. This results from the usage of a self stabilizing fundamental soliton in the intense arm of the fiber interferometer. We analysed splitting ratios of 91:09 and 85:15. Therefore the asymmetric NOLM, as described here, can be used in high speed telecommunication systems as an optical regenerator, without introducing a penalty regarding to the pulse shape and chirp. Therefore no changes in the fiber link design are needed which makes the NOLM a promising alternative to $3-\mathrm{R}$ regenerators, regarding performance and price.

\section{ACKNOWLEDGMENTS}

We would thank Arne Striegler from the Chair for Microwave Engineering and High Frequency Technology of the University Erlangen-Nuremberg for his support and the fruitful discussions. We would also like to greatfully acknowledge the financial support of the DFG. 


\section{REFERENCES}

[1] E.S. Awad, P.S. Cho, C. Richardson, N. Moulton, and J. Goldhar,"OOptical 3R regeneration with all-optical extraction and simultaneous wavelength conversion using a single ElectroAbsorbtion Modulator", Proc. ECOC, Copenhagen, Denmark, Vol. 3, 6.3.2, 2002

[2] G. Gavioli and P. Bayvel,"Novel, High-Stability 3R All Optical Regenerator Based On Polarization Switching in a Semiconductor Optical Amplifier", Proc. ECOC, Copenhagen, Denmark, Vol. 3, 7.3.2, 2002

[3] N.J. Smith and N.J. Doran, "Picosecond soliton transmission using concatenated nonlinear optical loop-mitror intensity filters",J. Opt. Soc. Am. B12, 1117-1125 (1995)

[4] R. Ludwig, A. Sizmann, U. Feiste, C. Schubert, M. Kroh, C.M. Weiner and H.G. Weber,"Experimental Verification of Noise Squeezing by an Optical Intensity Filter in HighSpeed Transmission", Proc. ECOC, Amsterdam, Netherlands, 2001

[5] M. Meissner, M. Roesch, B. Schmauss, G. Leuchs, "12dB of noise reduction by a NOLM based 2-R-regenerator",IEEE Photonics Technology Letters 15,1297-1299 (2003)

[6] R. Trebino, Frequency-Resolved Optical Gating: The Measurement of Ultrashort Laser Pulses(Kluwer Academic Publishers, 2002)

[7] M. Meissner, M. Roesch, B. Schmauss, N. Korolkova and G. Leuchs, “Optimum Splitting Ratio for amplifier noise reduction by an asymmetric nonlinear optical loop mirror", Proc. ECOC, Copenhagen, Denmark, Vol. 3, P3.08, 2002

[8] M. Meissner, M. Roesch, B. Schmauss and G. Leuchs, "Noise reduction performance of a NOLM based 2-R-regenerator in dependence on the OSNR", Proc. ECOC, Rimini, Italy, Vol. 3, We4P102, 2003

[9] G.P. Agrawal, Nonlinear Fiber Optics(Academic Press, 1995)

[10] P. Russell,“Photonic Crystal Fibers”, Science, Vol. 299, p.358, 2003 